\title{
Invited Editorial
}

\section{Making money in a downturn economy: Using the overshooting mechanism of stock prices for an investment strategy}

Received (in revised form): $22^{\text {nd }}$ November 2008

\begin{abstract}
Marco Folpmers
works for Capgemini Consulting in the Netherlands. The Matlab routine and data that are used in the article are available upon request. Marco Folpmers (1969) heads the Financial Risk Management consulting segment for Capgemini Consulting in the Netherlands. His expertise includes Basel II, especially economic capital, and financial modelling of complex financial instruments. He holds a Ph.D. in economics from the Free University of Amsterdam.
\end{abstract}

Correspondence: Capgemini, P.O. Box 2575, 3500 GN Utrecht, The Netherlands

E-mail: marco.folpmers@capgemini.com

ABSTRACT In this paper we show that a straightforward model to identify local extremes in a financial index can be applied to a subsequent period as a trading algorithm. The theoretical justification of the algorithm is the short-term behaviour of the index as an oscillation with predictable frequency. The economic interpretation of this behaviour relates to the overshooting and mean reversion properties of the financial time series. The algorithm is applied to the two main Euronext indices, sc. the AEX index and the CAC40 index. The algorithm outperforms the buy and hold investment strategy considerably in both cases.

Journal of Asset Management (2009) 10, 1-8. doi:10.1057/jam.2008.41

Keywords: asset management; algorithmic trading; financial time series modelling

\section{INTRODUCTION}

Financial markets have been hit hard by the credit crisis with stock prices declining heavily. In some markets there has been a vaporisation of more than 50 per cent of the market capitalisation since the mid-2007 peak. In this article, we will show that, notwithstanding this crisis, there are still investment opportunities provided that the buy and sell times are selected carefully. We will show how a straightforward algorithm can detect regularities in the timing of upswings and downswings and how a trading routine can capture the benefits of these moments of exit and entry. Before we explain the algorithm we will first go into some background about the basic ideas underlying the algorithm. After the algorithm's explanation, we will test its performance with the help of the two main regional indices traded at Euronext: the Amsterdam AEX index and the French CAC40 index.

\section{OVERSHOOTING AND MEAN REVERSION}

The underlying mechanism of the algorithm is the modelling of a financial time series as a 
short-term trendless oscillation with predictable frequency. The economic rationale of the algorithm can be explained by referring to the well-known overshooting and mean reversion properties of financial time series, which we apply here to index prices.

The idea of the overshooting behaviour of financial variables can be traced back to the seminal paper by Rudiger Dornbusch, who published his article 'Expectations and Exchange Rate Dynamics' in the Journal of Political Economy in 1976. ${ }^{1}$ His paper stated that exchange rates are expected to overshoot their long-term value after an increase in the money supply. An unanticipated permanent increase in the money supply leads to an initial fall in the interest rate and, hence, a depreciation of the exchange rate (using the theorem of uncovered interest rate parity). This depreciation is exaggerated, as the impact is enhanced by the foreign exchange market in which an increase in the money supply leads to a cheaper currency. When prices adjust to the new level of the money supply, and the real money supply $(\mathrm{M} / \mathrm{P})$ and the interest rate are back at their original values, the exchange rate appreciates to its long-term value (which is still below the initial value).

The phenomena of stock price overshooting, booms and crashes have often been attributed to outbursts of irrational speculation, 'manias' and 'panics'. This line of thought can be traced back to the famous economist J.K. Galbraith, whose 1954 bestseller The Great Crash, 1929 describes the Wall Street meltdown of stock prices of 1929 and the preceding speculative boom.

A specific overshooting theory for stock prices has been proposed by Joseph Zeira. ${ }^{2}$ He states that if fundamentals are changing until some future time, which is unknown in advance, prices go through a boom and a crash. His underlying mechanism is a Bayesian framework in which investors use prior information to assess the profitability of a stock. If the stock will now pay a dividend, the prior information is adjusted to higher expectations of profitability; and these expectations are reinforced each time the stock pays a dividend. Hence, the stock price rises to exaggerated levels. However, when dividends finally stop rising, the investors learn that the period of rising profits has come to an end and expectations for higher future dividends fall. As a consequence, the stock price is adjusted from its exaggerated level.

Overshooting behaviour is not always adjusted in a dramatic way by a burst of the bubble. Instead, we often witness mean reversion, or the tendency of financial values to adjust to a normal level after a short-term disequilibrium. Mean reversion is part of many interest rate models, Vasicek's model being one of the first to capture mean reverting interest rates. We think that mean reversion is a helpful concept for the understanding of short-term adjustments of stock prices.

In the next section we will discuss our stock price model and point out the parallels with the overshooting and mean reversion phenomena.

\section{THE ALGORITHM: EXPLOITATION OF IDENTIFIED LOCAL MINIMA AND MAXIMA}

From euronext.com we downloaded 2 years of historical daily AEX data running from 20 October 2006 until 17 October 2008. For our analysis we use opening prices (approximately 500 observations). The interesting feature of this specific historical window is that it contains both the upswing (the AEX was at 563 on 13 July 2007) and the subsequent downturn because of the subprime crisis below the 400 limit and even below the 300 frontier (the AEX was at 294 on 8 October 2008). On 17 October 2008 the AEX value was 259.95, which is 54 per cent lower than the peak of 563 , which occurred only 15 months earlier.

We will show how an algorithm that has been developed using the first half of the 
series as the development set can make a profit when applied to the second half of the set. The underlying driver is that commonalities exist within the entire series in terms of overshooting and mean reversion.

The basic idea behind the algorithm can be summarised as follows:

- The algorithm uses only the stock price index window of approximately 500 days. It does not use any other information.

- The time window is separated in a development set (first 210 days) and a test set (the rest of the time series). The development set equals one cycle in that the AEX index at the end of the first 210 days is back at its initial level.

- A minimum model and a maximum model are estimated with the help of the development set. This is the development set on which the parameters of the minimum and maximum models are calibrated. We will present the specifications of the minimum and maximum models below.

- The minimum and maximum models are applied to the test set to identify probable local minima and maxima.

- The initial liquidity balance equals $€ 1000$. For each trading day, a liquidity balance as well as the number of shares in the portfolio and their value using current prices are maintained.

- When a local minimum has been identified, the algorithm buys stocks at the current prices for a monetary amount of 40 per cent of the initial liquidity balance, that is $€ 400$. The liquidity balance decreases with $€ 400$ and the stocks bought are added to the portfolio.

- When a local maximum has been identified, the algorithm sells stocks at the current prices for a monetary amount of 40 per cent of the initial liquidity balance, that is $€ 400$. The liquidity balance increases with $€ 400$ and the stocks sold are subtracted from the portfolio.
- The entire portfolio is liquidated at the end of the period contained in the test set. The performance is assessed in terms of outperformance of a buy and hold strategy and the ratio of mean daily return and standard deviation of the daily returns (Sharpe ratio).

To prove robustness, the same algorithm has been applied to both main Euronext indices available, that is, the AEX and CAC40 indices.

Notice that the amount of stocks that are bought or sold whenever a local extreme has been identified is independent of the current liquidity balance and the current number of shares that are in the portfolio. That means that short selling is needed for an effective application of the algorithm.

We start with identifying the local extremes in the time series of the AEX index, which we call $I_{t}$. We define an indicator $L \_M_{i}$ for a local minimum at each moment in time $t$ as follows:

$$
\text { If } \begin{aligned}
& I_{t}<\min \left(I_{t-d 1}, I_{t-d 1+1}, \cdots, I_{t-1}\right) \\
& \quad \& I_{t}<\min \left(I_{t+1}, I_{t+2}, \cdots, I_{t+d 2}\right) \\
& \quad \rightarrow L_{-} \operatorname{Min}_{t}=1
\end{aligned}
$$

Else $L_{-} \operatorname{Min}_{t}=0$

The definition implies that a local minimum occurs at moment $t$ if the index value at that point in time is both lower than the minimum of the index values in the previous $d 1$ days and lower than the minimum of the index values in the following $d 2$ days after day $t$. Generally, for lower values of $d 1$ and $d 2$, we will identify more local minima than for higher values of $d 1$ and $d 2$.

In our approach, we have parameterised these coefficients as follows:

$$
d 1=d 2=3=d
$$

The local maxima have been defined in a parallel manner. The result is shown in Figure 1.

In this graph, the local minima have been marked with black boxes and the local maxima with red stars. We also show the 


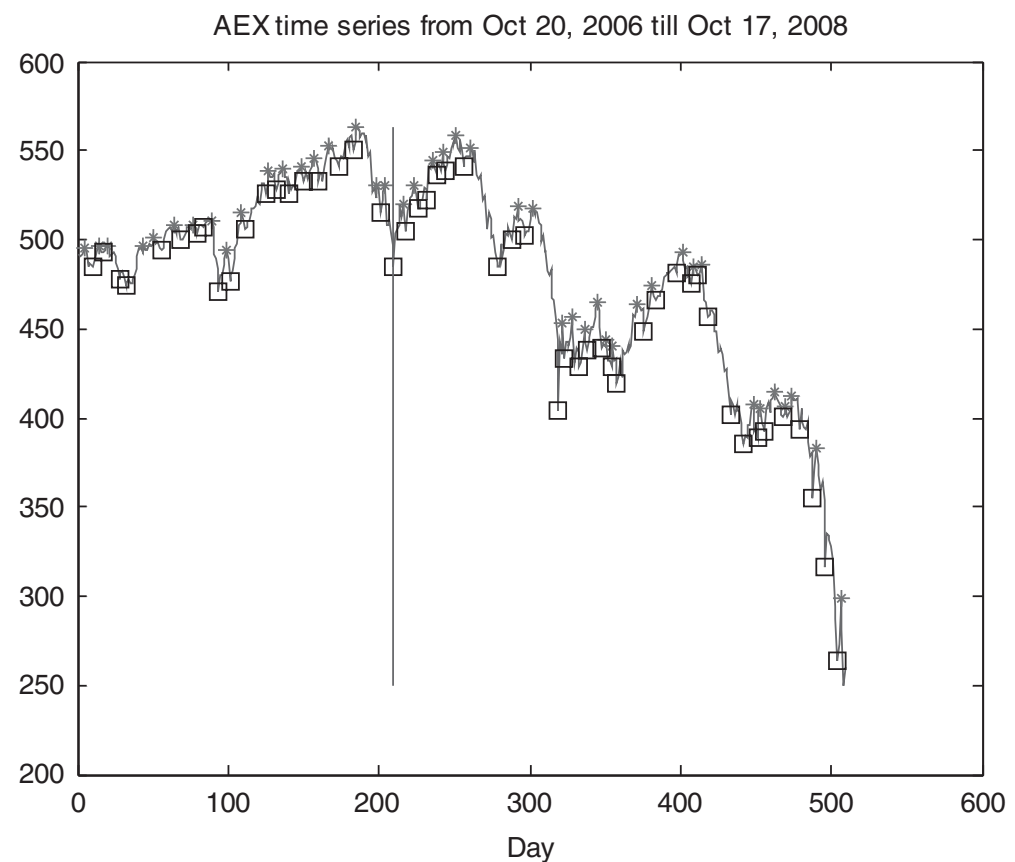

Figure 1: AEX with local minima (black boxes) and maxima (red stars), development set and test set.

separation between the development set (at the left of the vertical line) and the test set (at the right of the vertical line). What is interesting about this separation is that the vertical line is positioned in such a way that the big decline of the AEX index owing to the credit crisis is entirely in the test set. Our algorithm is trained on the development set and applied to the test set, a totally different regime.

At each time $t$ ( $t$ is in days), we compute the following attributes:

- The growth rate (GR) at time $t$ as compared to $t-1$;

- The number of successive increases (UPS) of the index up to time $t$;

- The number of successive decreases (DOWNS) of the index up to time $t$;

- The distance in days to the most recent local minimum (D_Min); if this distance is less than $d$, it is set to zero for reasons that we will explain below;

- The distance in days to the most recent local maximum (D_Max); again, if this distance is less than $d$, it is set to zero for reasons that we will explain below.
With the help of the development set we estimate a logit model for the probability $P \_$Max to have a local maximum at time $t$ :

$$
\begin{aligned}
& \operatorname{logit}\left(P_{-} \operatorname{Max}_{t}\right)=\log \left[\frac{P_{-} \operatorname{Max}_{t}}{1-P_{-} \operatorname{Max}_{t}}\right] \\
& =\beta_{0}+\beta_{1} \times G R_{t}+\beta_{2} \times U P S_{t} \\
& +\beta_{3} \times D_{-} M_{i n} \\
& +\beta_{4} \times D \_\operatorname{Max}_{t}+\varepsilon_{t}
\end{aligned}
$$

The minimum model for the probability $P \_$Min has the same specification with the only exception that it uses the independent variable DOWNS instead of UPS.

The coefficients, $t$-values and probabilities of these $t$-values are summarised in Table 1 for the minimum model. We see that the influence of the GR and the number of successive downs have a significant influence on the probability of encountering a local minimum. The coefficient of the GR has the expected negative sign in the minimum model.

An in-sample validity check is carried out by defining a cutoff point for the probability. If the estimated probability is higher than this 
Table 1: Coefficients, $t$-values and probabilities of the AEX index minimum model

AEX index

Minimum model

Model

Dependent variable: $L \_$Min

\begin{tabular}{lrrr}
\hline & \multicolumn{1}{c}{ Beta } & \multicolumn{1}{c}{$t$} & $P(\%)$ \\
\hline Intercept & -5.90 & -5.23 & 0.0 \\
GR & -77.42 & -2.47 & 1.3 \\
DOWNS & 1.64 & 4.38 & 0.0 \\
$D \_M i n$ & 0.05 & 0.87 & 38.7 \\
$D \_M a x$ & 0.07 & 1.09 & 27.6 \\
Performance & & & \\
$\quad$ Cutoff point & & & - \\
$\quad$ Percentage correctly & 87.0 & - & - \\
$\quad$ classified & & & \\
$\quad$ Percentage minimum & 100.0 & - & - \\
$\quad$ correct & & & \\
\hline
\end{tabular}

cutoff point, we conclude that the model has identified a minimum and the new variable, Model_Min ${ }_{t}$, is given the value 1 , otherwise it equals 0 . When comparing the minima identified by the minimum model with the real local minima (according to the definition above with $d=3$ ), the percentage correctly classified (PCC) equals 87.0 per cent, so

$$
P P C=P\left(\text { Model }_{-} \operatorname{Min}_{t}=L_{-} \operatorname{Min}_{t}\right)
$$

We have also calculated the percentage minimum correct $(P M C)$ that equals:

$$
\begin{aligned}
& P M C=P\left(\text { Model_Min } t_{t}\right. \\
& \left.=L_{-} M_{i n} \mid L_{-} \operatorname{Min}_{t}=1\right) \\
& =P\left(\text { Model }_{-} \text {Min }_{t}\right. \\
& \left.=1 \mid L_{-} \operatorname{Min}_{t}=1\right)
\end{aligned}
$$

We see that our in-sample validity is at a sufficiently high level to provide confidence in the model. The PCC and the PMC are illustrated in Figure 2. Again, we use black boxes for the local minima. We see that, with the cutoff value equal to 0.1 , we have identified all local minima. At the same time, we have some 'false-positives' in that we have high probabilities at a time $t$ that is not a local minimum. Generally, a higher cutoff point generates fewer false-positives. However, a higher cutoff point may fail to identify a local minimum. Based on these considerations during the in-sample validation, we have chosen the cutoff point to be set at 0.1 .

The minimum and maximum models have been estimated separately for the AEX index and the CAC40 index. In both cases we have only used the development set to obtain specifications for these models.

Subsequently, we have applied the specifications obtained to the test set to predict the occurrence of a local minimum or a local maximum. For each day $t$ in the development set, we have established the values of the independent variables: $G R$, number of successive ups (or downs for the minimum model), distance to last minimum and distance to last maximum. As we can only establish whether an observation is a local minimum or maximum with a time lag of 3 days (given the definition used above with $d 2=3$ ), the values for these distances are either 0 or equal to or larger than 3 . In this way, we only use information that is available at the start of day $t$.

As explained above, we use an initial liquidity balance equal to $€ 1000$. We invest $€ 400$ at a local minimum. At a local maximum, we sell $€ 400$ worth of stocks. At the end of the (development or test) period, the portfolio is liquidated and performance is determined. The algorithm's parameters such as $d 1, d 2$, the cutoff value $(0.10)$ and the tranche value (40 per cent of the initial liquidity is traded at each occurrence of an identified local minimum or maximum) have been established using the development set.

\section{PERFORMANCE}

Performance is measured in terms of absolute return over the period and outperformance when compared to a buy and hold strategy. The Sharpe ratio is also reported, which is defined as the ratio of the mean daily return divided by the standard deviation of the daily return. 


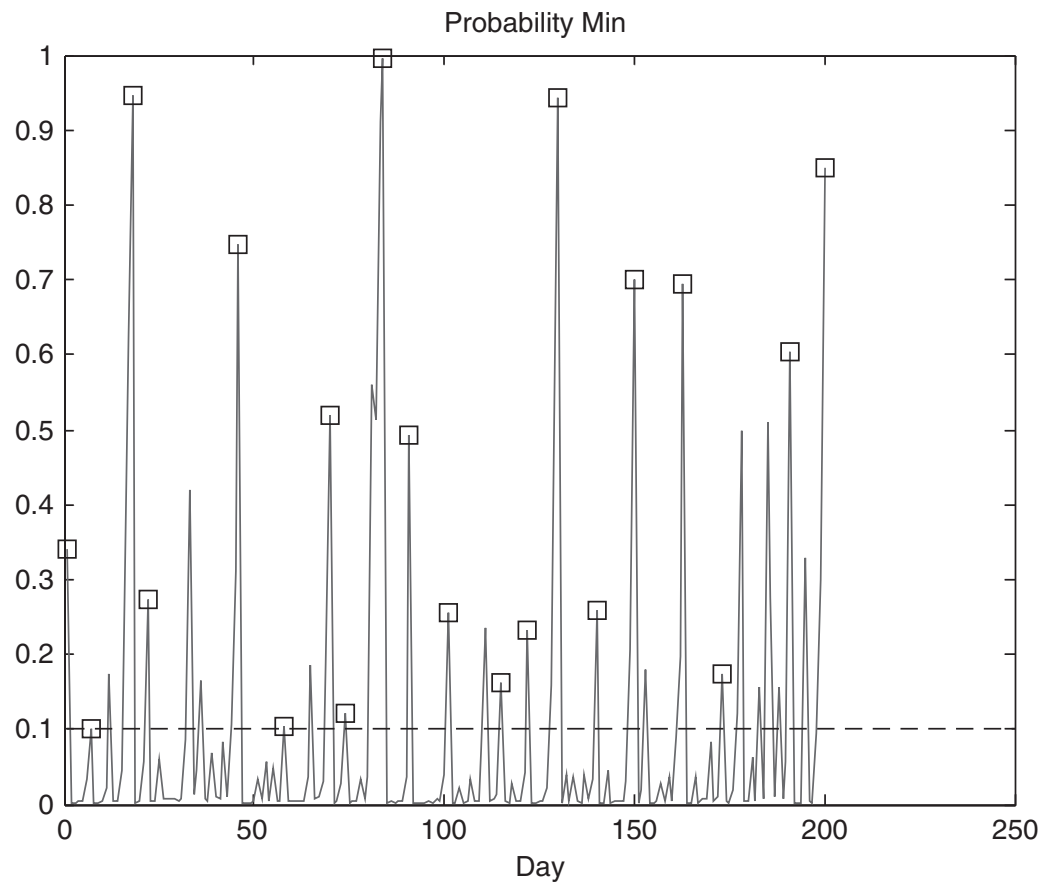

Figure 2: $P \_$Min for AEX index model, in-sample validity.

Table 2: Performance statistics

\begin{tabular}{|c|c|c|}
\hline & Buy and hold (\%) & Algo (\%) \\
\hline \multicolumn{3}{|c|}{$\begin{array}{l}\text { Absolute return over period } \\
\text { AEX }\end{array}$} \\
\hline In-sample & 0.2 & 52.9 \\
\hline Out-of-sample & -48.4 & 27.1 \\
\hline \multicolumn{3}{|l|}{ CAC40 } \\
\hline In-sample & -1.3 & 81.8 \\
\hline Out-of-sample & -38.3 & 121.7 \\
\hline \multicolumn{3}{|l|}{ Sharpe ratio } \\
\hline \multicolumn{3}{|l|}{ AEX } \\
\hline In-sample & 0.6 & 6.3 \\
\hline Out-of-sample & -9.0 & 4.1 \\
\hline \multicolumn{3}{|l|}{ CAC40 } \\
\hline In-sample & -0.1 & 8.4 \\
\hline Out-of-sample & -6.7 & 14.6 \\
\hline \multicolumn{3}{|l|}{ Outperformance } \\
\hline \multicolumn{3}{|l|}{ AEX } \\
\hline In-sample & 52.7 & \\
\hline Out-of-sample & 146.3 & \\
\hline \multicolumn{3}{|l|}{ CAC40 } \\
\hline In-sample & 84.2 & \\
\hline Out-of-sample & 259.3 & \\
\hline
\end{tabular}

The performance statistics are summarised in Table 2.

Notice that the in-sample absolute returns for the buy and hold strategy are almost zero, which is consistent with our definition of the test set: 210 days capturing an entire cycle.

We conclude that the algorithm is superior to the buy and hold strategy, especially during the stock market decline witnessed during the test period (see Figure 1), with outperformance statistics equal to 146 per cent for the AEX index and 259 per cent for the CAC40 index.

In Figure 3 we show the AEX test set and the local minima and maxima as identified by the minimum and maximum models.

Although these models have been estimated in the preceding period, they perform sufficiently well in this period to identify buy and sell points in time.

In Figure 4 the liquidity balance time series is shown along with the time series for the number of shares in the portfolio. We see that this number of shares is constant for the buy and hold strategy, as it buys approximately two shares for the initial liquidity of $€ 1000$. At the end of the period, we see this buy and hold strategy value reduced by almost a half, whereas the more 


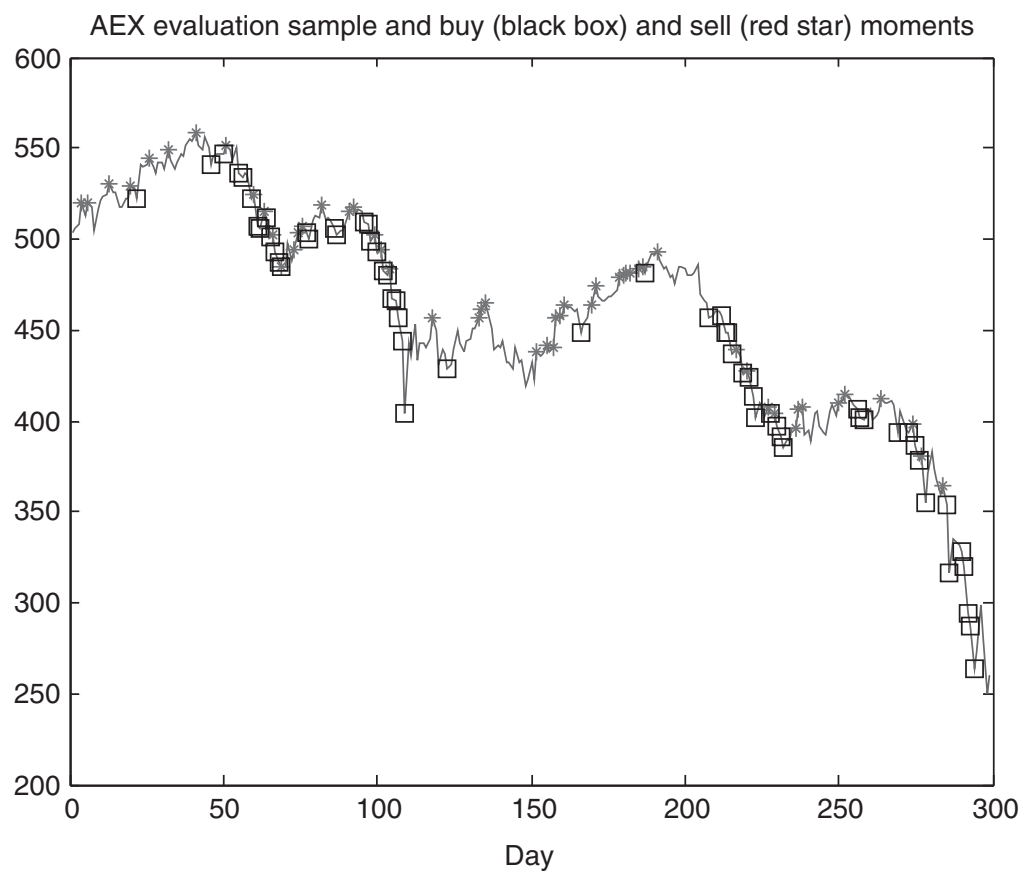

Figure 3: AEX test set, local minima and maxima as identified by minimum and maximum models.

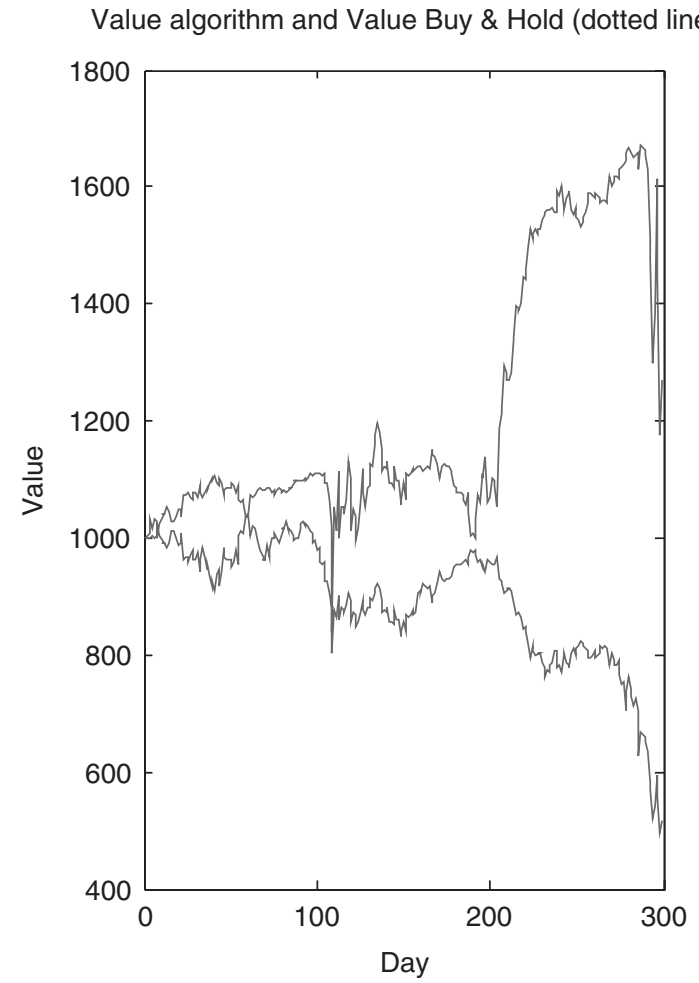

$\mathrm{Nr}$ shares algo and Buy \& Hold (dotted line)

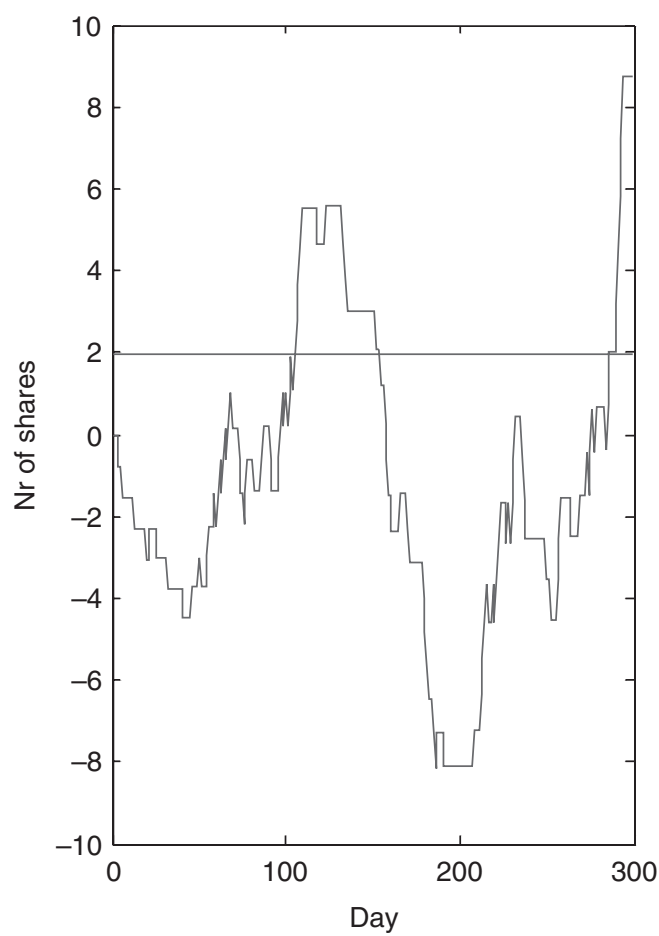

Figure 4: AEX index test set, time series of value (left panel) and number of shares in portfolio (right panel). 
active algorithm is able to make a profit of the decline.

In addition, Figure 4 shows clearly that naked short selling is absolutely necessary to have an effective deployment of the algorithm. Bans on naked short selling are anathema for the deployment of algorithms such as the one presented here. Although the algorithm is pretty straightforward (it uses only information that is contained in the series itself), it needs full flexibility in terms of naked short selling and negative liquidity (that is borrowing). The ban on naked short selling, such as the one the Dutch government issued on 21 September 2008 is not helpful indeed. ${ }^{3}$

As the algorithm is more active than the buy and hold strategy, it will also cause more transaction costs because of the higher number of transactions and the interest costs when borrowing. In this paper we abstract from these costs, as the absolute return figures shown in Table 2 are sufficiently high to cover all the additional transaction and interest costs.

The example presented here is applied to one index only. More sophisticated algorithms can be applied to long/short pairs or entire portfolios. These algorithms may also use more information than only the information that is contained in the series itself. Our example here is only meant to illustrate the power of modelling a stock price as an oscillation with a predictable frequency.

\section{A FREE LUNCH?}

Although this paper may seem to suggest it, we do not think that there is a free lunch. It takes qualified resources to generate the idea behind a promising algorithm, in addition to the entrepreneurship and capital required to exploit it. This exploitation can only be temporary so that fund managers will always keep a staff employed with the generation of new ideas and deployable algorithms.

We do not think that algorithms such as the one presented here exacerbate the crisis. As can be determined from Figure 4, the algorithm has a positive number of stocks in the portfolio between days 100 and 150 and at the end of the test period. As soon as stock prices are low enough, algorithms will identify a local minimum and try to profit from these low prices. This will not exacerbate the crisis at all. On the contrary, they will provide liquidity at times when distressed financial markets need it the most. For this reason, it is questionable whether a ban on naked short selling will prove to be a healthy policy instrument if it cripples algorithms such as the one presented here.

\section{ACKNOWLEDGMENTS}

I wish to thank Mr Parvez Patel who kindly revised a previous draft of this article.

\section{NOTES}

1. See Rogoff (2001) for a recent appreciation of Dornbusch's 1976 article.

2. See Zeira (1999).

3. See International Herald Tribune, 'Dutch ban naked short selling for 3 months', 21 September 2008, http:// www.iht.com/articles/ap/2008/09/21/business/EUNetherlands-Short-Selling.php. The week before, the US Securities and Exchange Commission banned all short selling in the shares of 799 financial companies until 2 October 2008. The United Kingdom and Germany have taken similar measures.

\section{REFERENCES}

Rogoff, K. (2001) Dornbusch's Overshooting Model after Twenty-five Years. Mundell-Fleming Lecture, November. Zeira, J. (1999) Informational overshooting, booms and crashes. Journal of Monetary Economics, 43(February): 237-257. 